\title{
$\begin{array}{r}\text { WAGENINGEN } \\ \hline \\ \hline\end{array}$
}

\section{Regulation of angiopoietin-like 4 and lipoprotein lipase in human adipose tissue}

Dijk, W., Schutte, S., Aarts, E. O., Janssen, I. M. C., Afman, L., \& Kersten, S.

This is a "Post-Print" accepted manuscript, which has been published in "J ournal of Clinical Lipidology"

This version is distributed under a non-commercial no derivatives Creative Commons (c) $(\mathcal{Q} \Theta \Theta(\mathrm{CC}-\mathrm{BY}-\mathrm{NC}-\mathrm{ND})$ user license, which permits use, distribution, and reproduction in any medium, provided the original work is properly cited and not used for commercial purposes. Further, the restriction applies that if you remix, transform, or build upon the material, you may not distribute the modified material.

Please cite this publication as follows:

Dijk, W., Schutte, S., Aarts, E. O., Janssen, I. M. C., Afman, L., \& Kersten, S. (2018). Regulation of angiopoietin-like 4 and lipoprotein lipase in human adipose tissue. Journal of Clinical Lipidology, 12(3), 773-783. DOI:

10.1016/j.jacl.2018.02.006

You can download the published version at:

https://doi.org/10.1016/j.jacl.2018.02.006 


\title{
Regulation of Angiopoietin-like 4 and Lipoprotein Lipase in Human Subcutaneous Adipose Tissue
}

\author{
Wieneke Dijk $^{1 *}$, Sophie Schutte ${ }^{1 *}$, Edo O. Aarts ${ }^{2}$, Ignace M.C. Janssen $^{2}$, Lydia Afman $^{1 *}$, \\ Sander Kersten ${ }^{1 *}$ \\ ${ }^{1}$ Nutrition, Metabolism and Genomics group, Division of Human Nutrition and Health, Wageningen \\ University, Stippeneng 4, 6708 WE, Wageningen, the Netherlands. ${ }^{2}$ Rijnstate Hospital and Vitalys \\ Clinics, Arnhem, the Netherlands.
}

To whom correspondence should be addressed: Sander Kersten, PhD, Nutrition, Metabolism and Genomics group, Division of Human Nutrition and Health, Stippeneng 4, 6708 WE Wageningen, The Netherlands, Email: sander.kersten@,wur.nl, Telephone: +31 317485787

* Authors contributed equally

Running title: ANGPTL4 and LPL in human adipose tissue

$\begin{array}{ll}\text { Abbreviations: } \\ \text { ANGPTL3 } & \text { Angiopoietin-like } 3 \\ \text { ANGPTL4 } & \text { Angiopoietin-like } 4 \\ \text { ANGPTL8 } & \text { Angiopoietin-like } 8 \\ \text { Endo H } & \text { Endoglycosidase H } \\ \text { LDLs } & \text { Low-density lipoproteins } \\ \text { LPL } & \text { Lipoprotein lipase } \\ \text { NEFA } & \text { Non-esterified fatty acids } \\ \text { PNGase F } & \text { Peptide:N-Glycosidase F } \\ \text { TG } & \text { Triglycerides } \\ \text { WAT } & \text { White adipose tissue }\end{array}$




\section{ABSTRACT}

Background: Elevated plasma triglycerides are increasingly viewed as a causal risk factor for coronary artery disease. One protein that raises plasma triglyceride levels and that has emerged as a modulator of coronary artery disease risk is angiopoietin-like 4 (ANGPTL4). ANGPTL4 raises plasma triglyceride levels by inhibiting lipoprotein lipase (LPL), the enzyme that catalyzes the hydrolysis of circulating triglycerides on the capillary endothelium.

Objective: The objective of the current study was to assess the association between ANGPTL4 and LPL in human adipose tissue, and to examine the influence of nutritional status on ANGPTL4 expression.

Methods: We determined ANGPTL4 and LPL mRNA and protein levels in different adipose tissue depots in a large number of severely obese patients who underwent bariatric surgery. Furthermore, in 72 abdominally obese subjects, we measured ANGPTL4 and LPL mRNA levels in subcutaneous adipose tissue in the fasted and post-prandial state.

Results: $A N G P T L 4$ mRNA levels were highest in subcutaneous adipose tissue, whereas $L P L$ mRNA levels were highest in mesenteric adipose tissue. ANGPTL4 and LPL mRNA levels were strongly positively associated in all three adipose tissue depots. In contrast, ANGPTL4 and LPL protein levels were negatively correlated in subcutaneous adipose tissue, suggesting a suppressive effect of ANGPTL4 on LPL protein abundance in subcutaneous adipose tissue. ANGPTL4 mRNA levels were $38 \%$ higher in the fasted compared to the post-prandial state.

Conclusion: Our data provide valuable insights into the relationship between ANGPTL4 and LPL in human adipose tissue, as well as the physiological function and regulation of ANGPTL4 in humans.

Keywords: Lipid metabolism, human adipose tissue, ANGPTL4, LPL, triglycerides. 


\section{INTRODUCTION}

Coronary artery disease is a major cause of morbidity and mortality worldwide ${ }^{1}$. Despite significant progress in the diagnosis, prevention and treatment of coronary artery disease, novel and effective treatments are needed to further reduce cardiovascular disease rates. Elevated plasma triglycerides are increasingly viewed as a causal risk factor for coronary artery disease ${ }^{2}$. Consequently, targeting plasma triglycerides may be a viable approach to lower coronary artery disease risk. One protein that regulates plasma triglyceride levels and that has recently emerged as a modulator of coronary artery disease risk is angiopoietin-like 4 (ANGPTL4). Specifically, studies have shown that carriers of the inactivating variant E40K in the ANGPTL4 gene are at reduced risk of developing coronary artery disease ${ }^{3,4}$. ANGPTL4 is a member of the angiopoietin-like protein family that also includes angiopoietin-like 3 (ANGPTL3) and angiopoietin-like 8 (ANGPTL8) ${ }^{5}$. ANGPTL4 likely modulates coronary artery disease risk by raising plasma triglyceride levels via inhibition of lipoprotein lipase (LPL), the enzyme that catalyzes the hydrolysis of circulating triglycerides on the capillary endothelium ${ }^{6}$. ANGPTL4 inhibits LPL by promoting the unfolding of LPL, which in turn leads to the dissociation of the catalytically active LPL dimer into inactive and unstable monomers ${ }^{7,8}$. Besides ANGPTL4, ANGPTL3 and ANGPTL8 also potently inhibit LPL activity and increase plasma triglyceride levels ${ }^{5}$. Notably, inactivation of ANGPTL3 via monoclonal antibodies and anti-sense oligonucleotides markedly reduces circulating levels of triglycerides and LDL cholesterol in humans ${ }^{9,10}$. Accordingly, ANGPTL3 and ANGPTL4 hold considerable promise as pharmacological targets for coronary artery disease.

As mentioned above, carriers of the E40K variant in the ANGPTL4 gene have significantly lower plasma triglyceride levels, as well as elevated HDL cholesterol levels. However, despite the strong genetic evidence for a role of ANGPTL4 in regulating plasma triglycerides 
in humans, the relation between circulating ANGPTL4 and plasma triglyceride levels remains somewhat ambiguous ${ }^{11-14}$. These and other observations have raised questions about the role of circulating ANGPTL4 in the regulation of LPL activity on the capillary endothelium. Indeed, several mouse studies indicate that locally expressed ANGPTL4 is important for regulating tissue LPL activity ${ }^{15-17}$. For example, the elevated expression of ANGPTL4 in adipose tissue during fasting likely accounts for the inhibition of adipose tissue LPL activity and the reduced uptake of TG-derived fatty acids in adipose tissue during fasting ${ }^{17,18}$. Moreover, we recently showed that, in mouse adipocytes, ANGTPL4 lowers the amount of LPL protein by promoting the intracellular degradation of mature glycosylated LPL ${ }^{19}$. Little is known about the regulation of ANGPTL4 mRNA and protein in human adipose tissue, and on its relationship with LPL. Accordingly, the objective of the current study was to assess the association between ANGPTL4 and LPL in human adipose tissue, by using material of different adipose tissue depots that was obtained from a large number of severely obese patients undergoing bariatric surgery. In addition, we investigated the relationship between ANGPTL3, ANGPTL4 and ANGPTL8 mRNA levels in liver material obtained from a subgroup of patients. Furthermore, we investigated the correlation between the above parameters and lipid and ANGPTL4 levels in blood plasma. Finally, in a separate study, we examined the influence of nutritional status on ANGPTL4, ANGPTL8, and LPL mRNA levels. 


\section{MATERIALS \& METHODS}

\section{MONDIAL study}

The MONDIAL study (acronym for Markers of Organ health in Non-diabetic and Diabetics; Intestine, Adipose tissue \& Liver) is a cross-sectional study in male and female patients undergoing bariatric surgery at Rijnstate hospital/ Vitalys clinics in Arnhem, the Netherlands. The study aimed to determine the health of adipose tissue depots, the liver, and the intestine, with a focus on examining potential differences in organ health between diabetics and nondiabetics, and to search for novel biomarkers for metabolic diseases. The collection of material was conducted in 15 patients in 2012 and in 61 patients in 2015 . Tissue samples were obtained from residual biological material from patients who underwent either a primary laparoscopic Roux-en-Y gastric bypass or a primary laparoscopic gastric sleeve procedure, deployed to induce weight loss and to alleviate co-morbidities of obesity. Both procedures were performed laparoscopically. Patients met the criteria for surgery from the Interdisciplinary European Guidelines for Surgery for Severe (Morbid) Obesity, amongst others aged 18-60, a BMI of over $40 \mathrm{~kg} / \mathrm{m}^{2}$ or a BMI between 35 and $40 \mathrm{~kg} / \mathrm{m}^{2}$ with comorbidity that is expected to improve after surgically-induced weight loss, a history of longstanding obesity ( $>5$ years), proven failed attempts to lose weight in a conventional way, or primarily successful weight loss with eventual weight regain, and the intention to adhere to a postoperative follow-up program ${ }^{20}$. For the MONDIAL study, we excluded non-Caucasian patients and targeted further recruitment of patients to obtain an equal ratio of females and males, as well as diabetics and non-diabetics. Patients were informed by their surgeon and one of the researchers about the study during a pre-operative visit two months prior to the surgical procedure, after which they could give a written acknowledgement of informed consent to participate. Participation included the consent that residual material would be used for 
scientific research and the withdrawal of a fasting blood sample before surgery. The blood sample was drawn prior to the administration of anesthetics on the operating table. Blood was collected in a $6 \mathrm{~mL}$ EDTA tube, after which the sample was centrifuged at $1500^{*} \mathrm{~g}$ at $4{ }^{\circ} \mathrm{C}$ to obtain plasma. Patients were informed that their decision to participate was totally voluntary and that they could withdraw at any time without giving a reason. Withdrawal would not affect their regular medical treatment. The study was approved by the local ethics committee of Rijnstate hospital.

\section{Bellyfat study}

The Bellyfat study was approved by the Medical Ethics Committee of Wageningen University and registered at ClinicalTrials.gov, identifier: NCT02194504. In short, 110 healthy participants aged $40-70$ years with abdominal obesity (BMI $>27 \mathrm{~kg} / \mathrm{m}^{2}$ or waist circumference $>88 \mathrm{~cm}$ for females, $>102 \mathrm{~cm}$ for males) were randomly assigned to one of three different energy-restricted diets. Here, we only report baseline data prior to the intervention. Subjects came to our department in the morning in the fasted state. After blood sampling and collection of a subcutaneous adipose tissue biopsy, subjects were given an in-house prepared mixed meal consisting of $76.3 \mathrm{~g}$ carbohydrates, $17.6 \mathrm{~g}$ protein, and $60.0 \mathrm{~g}$ fat. Four hours later, a second subcutaneous adipose tissue biopsy was taken, after making a new incision. The subcutaneous adipose tissue samples were obtained by needle biopsy from the periumbilical area under local anesthesia. The samples were rinsed to eliminate blood and were immediately frozen in liquid nitrogen. All samples were stored in aliquots at $-80^{\circ} \mathrm{C}$.

\section{RNA isolation \& $q P C R$}

Total RNA from residual material of the liver, the subcutaneous adipose tissue, the mesenteric adipose tissue and the omental adipose tissue from patients included in the MONDIAL study 
was isolated using TRIzol reagent (Life Technologies Europe BV, Bleiswijk, the Netherlands) and purified using the Qiagen RNeasy Mini kit (Qiagen, Venlo, the Netherlands). Quality of the isolated RNA was verified using an Agilent 2100 bioanalyzer (Agilent Technologies, Amsterdam, NL). Next, 500 ng of RNA was reverse transcribed using the First-Strand cDNA Synthesis Kit (Thermo Scientific, Landsmeer, the Netherlands). Real-time PCR was carried out using SensiMix (Bioline, GC Biotech, Alphen aan de Rijn, the Netherlands) on a CFX 384 Bio-Rad thermal cycler (Bio-Rad, Veenendaal, the Netherlands). Primer sequences can be found in Supplemental Table 1. 36B4 and BACTIN were used as housekeeping genes for the adipose tissue depots, whereas $C Y P A$ was used as housekeeping gene for the liver.

\section{Microarray analysis}

Purified RNA (100 ng) from subcutaneous adipose tissue of subjects included in the Bellyfat study was labeled with the Ambion WT expression kit (Invitrogen) and hybridized to an Affymetrix Human Gene 1.1 ST array plate (Affymetrix, Santa Clara, CA). Hybridization, washing, and scanning were carried out on an Affymetrix GeneTitan platform according to the instruction by the manufacturer. Analysis of microarray data was carried out as previously described ${ }^{21}$. The complete analysis of the microarray data from the Bellyfat study will be presented elsewhere.

\section{Western blots}

Protein lysates were made of residual material of the subcutaneous adipose tissue depot of patients included in the MONDIAL study and of whom sufficient material was available. Part of the material was lysed in RIPA lysis buffer $(25 \mathrm{mM}$ Tris- $\mathrm{HCl} \mathrm{pH} 7.6,150 \mathrm{mM} \mathrm{NaCl}, 1 \%$ NP-40, 1\% sodium; deoxycholate, $0.1 \%$ SDS; Thermo Scientific) supplemented with protease and phosphatase inhibitors (Roche, Woerden, The Netherlands) to make 30\% protein lysates. 
After a 30-minute incubation on ice, the lysates were spun down at $13.000 \mathrm{rpm}$ in order to get rid of non-dissolved material and fat. Following the transfer of the infranatant to a clean tube, this procedure was repeated twice to get rid of excess fat. Protein concentrations of lysates were determined with BCA reagent (Thermo Scientific) and subsequently adjusted to a concentration of $2.5 \mu \mathrm{g} / \mu \mathrm{L}$. Next, lysates were mixed with $2 \mathrm{x}$ LSB loading buffer and denatured at $95{ }^{\circ} \mathrm{C}$ for 5 minutes. For each patient, $5 \mu \mathrm{g}$ of protein was loaded per lane on 26wells Criterion $8-16 \%$ TGX gels (Bio-Rad) and separated by SDS gel electrophoresis. Separated proteins were transferred to a PVDF membrane by means of a Transblot Turbo System (Bio-Rad). Primary antibodies [goat anti-human LPL antibody (Santa Cruz Biotechnology, \#Y-20) or goat anti-LPL antibody (kind gift from Anne Beigneux, \#88B8 22); and rabbit anti-human ANGPTL4 antibody ${ }^{23}$ ) were used at a ratio of 1:750 (\#Y-20) or 1:1000 (\#88B8 and anti-human ANGPTL4) and incubated overnight at $4{ }^{\circ} \mathrm{C}$. Corresponding secondary antibodies (HRP-conjugated) (Sigma-Aldrich) were used at a 1:5000 dilution. Importantly, all samples were analyzed simultaneously to avoid variation. All incubations were done in Tris-buffered saline, $\mathrm{pH} 7.5$, with $0.1 \%$ Tween-20 (TBS-T) and 5\% dry milk, whereas all washing steps were done in TBS-T without dry milk. Blots were visualized using the ChemiDoc MP system (Bio-Rad) and Clarity ECL substrate (Bio-Rad). Quantification of bands was performed using ImageLab software (Bio-Rad). Of note, due to the absence of visual bands for ANGPTL4 $(n=1)$ and LPL $(n=2)$, protein data of three patients were excluded from subsequent statistical analyses.

\section{Plasma metabolites}

Plasma concentrations of triglycerides (Instruchemie, Delfzijl, the Netherlands) and free fatty acids (Wako Chemicals, Neuss, Germany; HR(2) Kit) in fasted plasma samples of patients included in the MONDIAL study were determined following the manufacturers' instructions. 
Plasma concentrations of glucose were determined on the morning of the operation using Accu-Chek meters (Roche). Plasma ANGPTL4 levels were determined as described previously ${ }^{24}$ using an anti-human ANGPTL4 polyclonal goat IgG antibody (AF3485; R\&D Systems, Abingdon, United Kingdom). Briefly, 96-wells plates were coated with antiANGPTL4 antibody and incubated overnight at $4{ }^{\circ} \mathrm{C}$. The next day, $100 \mu \mathrm{L}$ of 20 -fold diluted human plasma was applied and incubated at room temperature for 2 hours. Next, $100 \mu \mathrm{L}$ of biotinylated anti-human ANGPTL4 polyclonal goat IgG antibody (BAF3485; R\&D Systems) was added to each well and incubated at room temperature for 2 hours, followed by the addition of streptavidin-conjugated HRP for $20 \mathrm{~min}$. Tetramethylbenzidine substrate reagent was added for 6 min, where after the reaction was stopped by adding $50 \mu \mathrm{L}$ of $10 \% \mathrm{H}_{2} \mathrm{SO}_{4}$. The absorbance was measured at $450 \mathrm{~nm}$.

\section{Statistical analyses}

Statistical analyses were performed using GraphPad software (La Jolla, California, United States). A Kruskal-Wallis test followed by a Dunn's test was used to compare ANGPTL4 and LPL expression values in different adipose tissue depots. To determine the correlation coefficients between multiple parameters, we employed non-parametric Spearman's correlations. P-values of $<0.05$ were considered statistically significant. Differences in $(\log -$ transformed) subcutaneous adipose tissue gene expression between the fasted and postprandial state were evaluated using a paired Student's t-test. 


\section{RESULTS}

Patient's characteristics of the MONDIAL study

Patient characteristics of the MONDIAL study are shown in Table 1. Age of the participants varied between 21 and 69 years old. Participants were almost equally distributed among men (46\%) and women (54\%). The mean body weight and BMI were $129.0 \pm 18.9 \mathrm{~kg}$ and $42.7 \pm$ $5.3 \mathrm{~kg} / \mathrm{m}^{2}$, respectively (means $\pm \mathrm{SD}$ ). The mean fasting plasma glucose concentration was $7.8 \pm 2.8 \mathrm{mmol} / \mathrm{Liter}$.

mRNA levels of ANGPTL3, ANGPTL4 and ANGPTL8 are not correlated in human liver ANGPTL4 is abundantly secreted into the circulation by the liver, where its expression is regulated by the transcription factor PPARA ${ }^{25}$. Interestingly, in the human liver material, no correlation was found between the mRNA levels of ANGPTL4 and PPARA (Figure 1A). Also, no correlation was found between mRNA levels of ANGPTL4 and ANGPTL3 or $A N G P T L 8$ (Figure 1B). The correlations between the gene expression levels of ANGPTL3, ANGPTL4, ANGPTL8 and PPARA with various plasma parameters are depicted in Figure 1C. Notably, no significant correlation was found between hepatic ANGPTL4 mRNA levels and the plasma ANGPTL4 concentration or between hepatic ANGPTL4 mRNA levels and the plasma triglyceride concentration (Figure 1C, Supplemental Figure 1A \& 1B).

Expression levels of ANGPTL4 are highest in the subcutaneous adipose tissue

Expression of ANGPTL4 in human and mouse adipose tissue is comparatively high ${ }^{17,18,25}$. Interestingly, in our study, ANGPTL4 mRNA levels were significantly higher in the subcutaneous adipose tissue depot than in the omental and mesenteric adipose tissue depots (Figure 2A). In contrast, mRNA levels of $L P L$ were highest in the mesenteric adipose tissue depot (Figure 2B). 
Strikingly, we observed a highly significant correlation between ANGPTL4 mRNA levels in the subcutaneous adipose tissue depot and ANGPTL4 mRNA levels in the omental and mesenteric adipose tissue depots (Figure 2C). Similarly, the $L P L$ mRNA levels in the subcutaneous adipose tissue were significantly correlated with $L P L$ mRNA in the omental and mesenteric adipose tissue depots (Figure 2D).

Expression levels of ANGPTL4 and LPL are positively correlated in the omental and subcutaneous adipose tissue.

ANGPTL4 potently inhibits LPL in the adipose tissue of mice, but data on the relationship between ANGPTL4 and LPL expression in human adipose tissue are scarce ${ }^{17}$. We observed a highly significant, positive correlation between ANGPTL4 mRNA levels and LPL mRNA levels in the omental and subcutaneous adipose tissue depots (Figure 3A and Figure 3B), but not in the mesenteric adipose tissue depot (Figure 3C). Given that both LPL and ANGPTL4 are under positive control of the transcription factor PPAR $\gamma$, we examined the association between the mRNA levels of $A N G P T L 4$ and $L P L$, respectively, and PPARG mRNA levels in the different adipose tissue depots ${ }^{6,25,26}$. Whereas ANGPTL4 mRNA levels in the various fat depots showed only a weak or no correlation with PPARG mRNA (Figure 3D; Supplemental Figure 2A), $L P L$ mRNA levels were strongly and significantly correlated with PPARG mRNA levels in all three adipose tissue depots (Figure 3D, Supplemental Figure 2B). An overview of the correlations between $A N G P T L 4, L P L$, and PPARG mRNA levels in the three different adipose tissue depots, as well as various plasma parameters, is shown in Figure 3D. Specifically, we found no association between the mRNA levels of ANGPTL4 in the subcutaneous, omental and mesenteric adipose tissue depots and the plasma ANGPTL4 concentration or plasma triglyceride concentration (Figure 3D, Supplemental Figure 1C \& 1D). 
Protein levels of LPL and ANGPTL4 are negatively correlated in subcutaneous adipose tissue To determine the relationship between the protein levels of LPL and ANGPTL4 in human adipose tissue, we measured LPL and ANGPTL4 protein in the subcutaneous adipose tissue by Western blot using validated antibodies against ANGPTL4 and LPL (Supplemental Figures 3 \& 4). As previously shown, ANGPTL4 was only detectable in human adipose tissue as the full-length protein ${ }^{23}$. After quantification, a significant negative correlation was observed between ANGPTL4 and LPL protein level $(\mathrm{R}=-0.2314, \mathrm{p}=0.0461)$ (Figure 4, Supplemental Figure 4). We also attempted to determine the levels of mature glycosylated LPL by means of treatment of the adipose tissue lysates with the endoglycosidase Endo $\mathrm{H}$, an enzyme that removes high mannose carbohydrates from glycosylated asparagine residues and that is thus expected to act upon immature, but not mature glycosylated LPL. Unfortunately, in contrast to mouse adipose tissue and previously published data ${ }^{19}$, no reduction in LPL weight upon treatment with Endo $\mathrm{H}$ was detected, suggesting that most of the LPL found in the human subcutaneous adipose tissue is in the mature glycosylated form (Supplemental Figure 5) ${ }^{27-30}$.

ANGPTL4 mRNA levels are higher in the fasted than the post-prandial state in subcutaneous adipose tissue

ANGPTL4 was initially cloned as the Fasting-Induced Adipose Factor ${ }^{25}$. While the induction of ANGPTL4 mRNA and protein by fasting in adipose tissue is evident in mouse studies $17,18,25$, it is unclear whether ANGPTL4 is also upregulated by fasting in human adipose tissue. To answer that question, we measured ANGPTL4 mRNA in subcutaneous adipose tissue biopsies taken from 72 human subjects after an overnight fast and 4 hours after receiving a mixed meal. ANGPTL4 mRNA levels were $38 \%$ higher in the fasted state compared to the 
post-prandial state $\left(\mathrm{P}<1 * 10^{-15}\right)$ (Figure 5). By contrast, mRNA levels of ANGPTL8 were $64 \%$ lower in the fasted state compared to the post-prandial state $\left(\mathrm{P}<1 * 10^{-22}\right)$ (Figure 5). $L P L$ mRNA levels were not significantly different between the fasted and post-prandial state (Figure 5). These data indicate that ANGPTL4 expression in human adipose tissue is induced by fasting, whereas $A N G P T L 8$ expression is reduced by fasting. 


\section{DISCUSSION}

This study was conducted to clarify the regulation of ANGPTL4 and LPL in human adipose tissue using residual material of omental, mesenteric and subcutaneous adipose tissue obtained from a large cohort of severely obese patients undergoing bariatric surgery. In addition, we aimed to examine the influence of nutritional status on ANGPTL4 mRNA levels in human subcutaneous adipose tissue. ANGPTL4 mRNA levels were highest in the subcutaneous adipose tissue depot, whereas $L P L$ mRNA levels were highest in the mesenteric adipose tissue depot. $A N G P T L 4$ and $L P L$ mRNA levels were strongly positively associated in all three adipose tissue depots. In contrast, ANGPTL4 and LPL protein levels in subcutaneous adipose tissue were negatively correlated. Finally, consumption of a mixed meal significant reduced ANGPTL4 mRNA levels in human subcutaneous adipose tissue.

Consistent with earlier observations in mouse adipose tissue ${ }^{17,18,25}$ our results for the first time demonstrate that fasting increases $A N G P T L 4$ mRNA expression in human adipose tissue. Studies using mouse models have demonstrated that the upregulation of ANGPTL4 in adipose tissue during fasting leads to a reduction in local LPL activity and a concomitant decrease in the hydrolysis of circulating triglycerides ${ }^{17,18,31}$. As a consequence, circulating lipids are diverted away from storage in adipose tissue to other tissues. Based on the data presented here, a similar role can be envisioned for ANGPTL4 in human adipose tissue.

Previously, we demonstrated that ANGPTL4 decreases the levels of LPL protein in mouse adipose tissue, which is likely achieved by promoting the intracellular degradation of LPL ${ }^{19}$. Here, we find a significant negative correlation between ANGPTL4 and LPL protein in human subcutaneous adipose tissue. It is reasonable to suggest that this negative correlation may be a reflection of the stimulatory effect of ANGPTL4 on LPL degradation, which would 
imply that ANGPTL4 regulates LPL protein abundance in human adipose tissue as well. To further investigate the suppressive effect of ANGPTL4 on LPL protein abundance in human fat, it would have been very worthwhile to be able to study the effect of feeding status on ANGPTL4 and LPL protein levels in human subcutaneous adipose tissue. Unfortunately, not enough adipose tissue samples were left to measure ANGPTL4 and LPL protein levels. Also, given the unhealthy metabolic phenotype of the patients included in the MONDIAL study, the negative correlation between ANGPTL4 and LPL protein levels requires confirmation in adipose tissue of lean individuals.

In our in vitro and mouse studies we observed that ANGPTL4 specifically reduced the level of mature glycosylated, but not immature glycosylated LPL ${ }^{19}$. Surprisingly, only mature glycosylated LPL could be detected in human adipose tissue. In addition, the migration of LPL was only modestly reduced following the removal of all asparagine-linked glycosylation by PNGase F, suggesting that LPL in the subcutaneous adipose tissue of our patients was only glycosylated at one of the two potential glycosylation sites ${ }^{32}$ (Supplemental Figure 4). These data are in disagreement with previously published studies showing that LPL in human adipose tissue has two glycosylation chains that are primarily of the immature kind ${ }^{28-30}$. Further studies into the exact nature of the glycosylation side chains of human adipose tissue LPL, by using endoglycosidases with different specificities such as Endo F1, Endo F2 or PNGase A, might provide an explication for this discrepancy ${ }^{28-30}$.

In contrast to the negative correlation between adipose tissue ANGPTL4 and LPL at the protein level, we observed a strong positive correlation between ANGPTL4 and LPL at the gene expression level. The positive correlation between ANGPTL4 and LPL mRNA may be due to a common transcription factor that drives the expression of both genes. One candidate 
is PPARG, as both $L P L$ and ANGPTL4 are established PPARG target genes in adipose tissue 25,26,33. Yet, despite a highly significant correlation between $L P L$ and PPARG mRNA levels, we only found a modest correlation between ANGPTL4 and PPARG mRNA. An alternative candidate is the glucocorticoid receptor. Indeed, glucocorticoids have been shown to increase $L P L$ mRNA in human adipose organ cultures ${ }^{34}$ and to upregulate ANGPTL4 mRNA in human primary adipocytes ${ }^{35}$.

Our study indicated that ANGPTL4 mRNA levels were highest in the subcutaneous adipose tissue depot, whereas $L P L$ mRNA levels were highest in the mesenteric adipose tissue depot. Consequently, the ratio of $L P L$ to $A N G P T L 4$ mRNA was highest in the mesenteric fat. A high ratio of LPL to ANGPTL4 is expected to favor fat storage. The molecular basis for the difference in absolute ANGPTL4 mRNA levels between the fat depots is unclear, but might be related to differences in oxygen levels or inflammatory status between the depots, as hypoxia and inflammatory mediators have been shown to influence ANGPTL4 mRNA levels ${ }^{36-39}$. Interestingly, we observed a clear positive correlation in ANGPTL4 mRNA levels between the different adipose tissue depots, suggesting that subject-specific mechanisms driving ANGPTL4 mRNA expression are active across the three fat depots. Whether the above observations can be generalized to lean individuals or are specific to obese individuals remains unclear.

In our study, we failed to observe a significant positive correlation between the concentration of ANGPTL4 in plasma and ANGPTL4 mRNA levels in liver and adipose tissue. These data suggest that the plasma concentration of ANGPTL4 is not primarily driven by hepatic and/or adipose ANGPTL4 mRNA levels. Previously, we found that the liver-specific activation of ANGPTL4 transcription by the PPAR $\alpha$ agonist fenofibrate increased plasma ANGPTL4 
concentrations in human subjects ${ }^{24}$. These data suggest that changes in ANGPTL4 production in human liver can influence plasma ANGPTL4 concentrations. In a crosssectional study, however, other factors may overpower the influence of tissue-specific ANGPTL4 mRNA levels on the plasma ANGPTL4 concentration.

ANGPTL4 and the related ANGPTL3 and ANGPTL8 share a common structure and the capacity to regulate LPL activity and plasma triglycerides, but to what extent their physiological functions are interconnected remains unclear ${ }^{5,40-42}$. Our data indicate that ANGPTL3, ANGPTL4 and ANGPTL8 expression levels are differentially regulated in human liver, as we did not observe a significant correlation between liver ANGPTL4 mRNA levels and liver $A N G P T L 3$ and $A N G P T L 8$ mRNA levels. These data corroborate previous studies showing that ANGPTL4 mRNA, and ANGPTL 8 mRNA in liver are oppositely regulated by insulin and during physiological conditions such as fasting and refeeding ${ }^{43-45}$. Furthermore, it has been demonstrated that ANGPTL4 is an established PPARA target gene in the liver, whereas $A N G P T L 3$, and possibly also $A N G P T L 8$, are LXR target genes ${ }^{25,46,47}$. Strikingly, we found that, opposite to ANGPTL4, ANGPTL8 mRNA in human adipose tissue is very significantly increased upon feeding. These data are consistent with previous data showing that ANGPTL8 mRNA in human adipose tissue is highly induced by insulin ${ }^{48}$. Taken together, the differential regulation and lack of association between ANGPTL4 mRNA and ANGPTL3/ANGPTL8 mRNA suggests that the corresponding proteins likely impact plasma LPL activity and plasma triglyceride levels during different physiological conditions ${ }^{5}$.

In conclusion, we report a negative correlation between ANGPTL4 and LPL protein levels in human subcutaneous adipose tissue, which might reflect a suppressive effect of ANGPTL4 on LPL protein abundance. In addition, we found that adipose tissue ANGPTL4 mRNA levels 
were significantly lower in the fed state compared to the fasted state. These data provide valuable insights into the physiological function of ANGPTL4 in humans.

\section{Acknowledgements}

The authors would like to thank Ieke van der Wel, Berit Follong and Mechteld Grootte Bromhaar for their valuable work during the MONDIAL study, as well as Karin Mudde for help with the ANGPTL4 ELISA. We also kindly thank Anne Beigneux and Katsuyuki Nakajima for providing the goat anti-human LPL antibody 88B8.

This work was supported by grant 12CVD04 from the Fondation Leducq.

\section{Conflict of interest}

None of the authors have a potential conflict of interest to report relevant to this study.

\section{Author's contribution}

S.S., E.A., I.M.C.J. and L.A. conceived and executed the MONDIAL study. S.S. and L.A. conceived and executed the Bellyfat study. S.S. collected material during the MONDIAL and the Bellyfat study. W.D. and S.K conceived the idea for the study described in this paper, with input from S.S. and L.A. W.D. performed most experiments, with help of S.S. W.D. and S.K performed data analysis and wrote the initial draft of the paper. All authors approved the final draft of this article. 


\section{References}

1. World Health Organization (WHO). Cardiovascular diseases (CVDs) Fact Sheet 317. http://www.who.int/mediacentre/factsheets/fs317/en/. Published 2017. Accessed July 30, 2017.

2. Miller M, Stone NJ, Ballantyne C, et al. Triglycerides and cardiovascular disease: a scientific statement from the American Heart Association. Circulation. 2011;123(20):2292-2333. doi:10.1161/CIR.0b013e3182160726.

3. Dewey FE, Gusarova V, O'Dushlaine C, et al. Inactivating Variants in ANGPTL4 and Risk of Coronary Artery Disease. N Engl J Med. 2016;374(12):1123-1133. doi:10.1056/NEJMoa1510926.

4. Myocardial Infarction Genetics and CARDIoGRAM Exome Consortia Investigators, Stitziel NO, Stirrups KE, et al. Coding Variation in ANGPTL4, LPL, and SVEP1 and the Risk of Coronary Disease. N Engl J Med. 2016;374(12):1134-1144. doi:10.1056/NEJMoa1507652.

5. Dijk W, Kersten S. Regulation of lipid metabolism by angiopoietin-like proteins. Curr Opin Lipidol. 2016;27(3). doi:10.1097/MOL.0000000000000290.

6. Kersten S. Physiological regulation of lipoprotein lipase. Biochim Biophys Acta. 2014;1841(7):919-933. doi:10.1016/j.bbalip.2014.03.013.

7. Sukonina V, Lookene A, Olivecrona T, Olivecrona G. Angiopoietin-like protein 4 converts lipoprotein lipase to inactive monomers and modulates lipase activity in adipose tissue. Proc Natl Acad Sci U S A. 2006;103(46):17450-17455. doi:10.1073/pnas.0604026103.

8. Mysling S, Kristensen KK, Larsson M, et al. The angiopoietin-like protein ANGPTL4 catalyzes unfolding of the hydrolase domain in lipoprotein lipase and the endothelial membrane protein GPIHBP1 counteracts this unfolding. Elife. 2016;5:18. doi:10.7554/eLife.20958.

9. Graham MJ, Lee RG, Brandt TA, et al. Cardiovascular and Metabolic Effects of ANGPTL3 Antisense Oligonucleotides. N Engl J Med. 2017;377(3):222-232. doi:10.1056/NEJMoa1701329.

10. Dewey FE, Gusarova V, Dunbar RL, et al. Genetic and Pharmacologic Inactivation of ANGPTL3 and Cardiovascular Disease. N Engl J Med. 2017;377(3):211-221. doi:10.1056/NEJMoa1612790.

11. Mehta N, Qamar A, Qu L, et al. Differential association of plasma angiopoietin-like proteins 3 and 4 with lipid and metabolic traits. Arterioscler Thromb Vasc Biol. 2014;34(5):1057-1063. doi:10.1161/ATVBAHA.113.302802.

12. Robciuc MR, Tahvanainen E, Jauhiainen M, Ehnholm C. Quantitation of serum angiopoietin-like proteins 3 and 4 in a Finnish population sample. J Lipid Res. 2010;51(4):824-831. doi:10.1194/j1r.M002618.

13. Robciuc MR, Naukkarinen J, Ortega-Alonso A, et al. Serum angiopoietin-like 4 protein levels and expression in adipose tissue are inversely correlated with obesity in monozygotic twins. J Lipid Res. 2011;52(8):1575-1582. doi:10.1194/jlr.P015867.

14. Smart-Halajko MC, Robciuc MR, Cooper JA, et al. The relationship between plasma angiopoietin-like protein 4 levels, angiopoietin-like protein 4 genotype, and coronary heart disease risk. Arterioscler Thromb Vasc Biol. 2010;30(11):2277-2282. doi:10.1161/ATVBAHA.110.212209.

15. Dijk W, Heine M, Vergnes L, et al. ANGPTL4 mediates shuttling of lipid fuel to brown adipose tissue during sustained cold exposure. Elife. 2015;4. doi:10.7554/eLife.08428.

16. Catoire M, Alex S, Paraskevopulos N, et al. Fatty acid-inducible ANGPTL4 governs 
lipid metabolic response to exercise. Proc Natl Acad Sci U S A. 2014;111(11):E104352. doi:10.1073/pnas.1400889111.

17. Kroupa O, Vorrsjö E, Stienstra R, et al. Linking nutritional regulation of Angpt14, Gpihbp1, and Lmf1 to lipoprotein lipase activity in rodent adipose tissue. BMC Physiol. 2012;12(1):13. doi:10.1186/1472-6793-12-13.

18. Cushing EM, Chi X, Sylvers KL, Shetty SK, Potthoff MJ, Davies BSJ. Angiopoietinlike 4 directs uptake of dietary fat away from adipose during fasting. Mol Metab. 2017;6(8):809-818. doi:10.1016/j.molmet.2017.06.007.

19. Dijk W, Beigneux AP, Larsson M, Bensadoun A, Young SG, Kersten S. Angiopoietinlike 4 (ANGPTL4) promotes intracellular degradation of lipoprotein lipase in adipocytes. J Lipid Res. 2016;58(12):7250-7257. doi:10.1194/jlr.M067363.

20. Fried M, Hainer V, Basdevant A, et al. Interdisciplinary European Guidelines on Surgery of Severe Obesity. Obes Facts. 2008;1(1):52-59. doi:10.1159/000113937.

21. Janssen AWF, Betzel B, Stoopen G, et al. The impact of PPAR $\alpha$ activation on whole genome gene expression in human precision cut liver slices. BMC Genomics. 2015;16(1):760. doi:10.1186/s12864-015-1969-3.

22. Allan CM, Larsson M, Hu X, et al. An LPL-specific monoclonal antibody, 88B8, that abolishes the binding of LPL to GPIHBP1. J Lipid Res. 2016;57(10):1889-1898. doi:10.1194/jlr.M070813.

23. Mandard S, Zandbergen F, Tan NS, et al. The direct peroxisome proliferator-activated receptor target fasting-induced adipose factor (FIAF/PGAR/ANGPTL4) is present in blood plasma as a truncated protein that is increased by fenofibrate treatment. J Biol Chem. 2004;279(33):34411-34420. doi:10.1074/jbc.M403058200.

24. Kersten S, Lichtenstein L, Steenbergen E, et al. Caloric restriction and exercise increase plasma ANGPTL4 levels in humans via elevated free fatty acids. Arterioscler Thromb Vasc Biol. 2009;29(6):969-974. doi:10.1161/ATVBAHA.108.182147.

25. Kersten S, Mandard S, Tan NS, et al. Characterization of the fasting-induced adipose factor FIAF, a novel peroxisome proliferator-activated receptor target gene. J Biol Chem. 2000;275(37):28488-28493. doi:10.1074/jbc.M004029200.

26. Yoon JC, Chickering TW, Rosen ED, et al. Peroxisome proliferator-activated receptor gamma target gene encoding a novel angiopoietin-related protein associated with adipose differentiation. Mol Cell Biol. 2000;20(14):5343-5349. doi:10.1128/MCB.20.14.5343-5349.2000.Updated.

27. Fager G, Semb H, Enerbäck S, et al. Hyperlipoproteinemia type I in a patient with active lipoprotein lipase in adipose tissue and indications of defective transport of the $\begin{array}{llll}\text { enzyme. } & \text { J } & \text { Lipid } & \text { Res. }\end{array}$ http://www.ncbi.nlm.nih.gov/pubmed/2205700.

28. Sakayama K, Masuno H, Okumura H, Shibata T, Okuda H. Glycosylation of lipoprotein lipase in human subcutaneous and omental adipose tissues. Biochim Biophys Acta - Lipids Lipid Metab. 1992;1127:153-156. doi:10.1016/00052760(92)90271-V.

29. Sakayama K, Masuno H, Kidani T, Matsuda Y, Yamamoto H, Okuda H. Synthesis of active high mannose-type lipoprotein lipase in human adipose tissues. Atherosclerosis. 2001;155:29-35. doi:10.1016/S0021-9150(00)00525-6.

30. Park JW, Yang JY, Rhee SR, et al. Glycosylation of lipoprotein lipase in human subcutaneous lipomas. Horm Metab Res. 1996;28(1):7-10. doi:10.1055/s-2007979120.

31. Lichtenstein L, Berbée JFP, van Dijk SJ, et al. Angptl4 upregulates cholesterol synthesis in liver via inhibition of LPL- and HL-dependent hepatic cholesterol uptake. Arterioscler Thromb Vasc Biol. 2007;27(11):2420-2427. 
doi:10.1161/ATVBAHA.107.151894.

32. Semenkovich CF, Luo CC, Nakanishi MK, Chen SH, Smith LC, Chan L. In vitro expression and site-specific mutagenesis of the cloned human lipoprotein lipase gene. Potential N-linked glycosylation site asparagine 43 is important for both enzyme activity and secretion. J Biol Chem. 1990;265(10):5429-5433. http://www.ncbi.nlm.nih.gov/pubmed/2318821.

33. Schoonjans K, Peinado-Onsurbe J, Lefebvre AM, et al. PPARalpha and PPARgamma activators direct a distinct tissue-specific transcriptional response via a PPRE in the lipoprotein lipase gene. EMBO J. 1996;15(19):5336-5348. http://www.pubmedcentral.nih.gov/articlerender.fcgi?artid $=452277 \&$ tool=pmcentrez $\&$ rendertype $=$ abstract.

34. Fried SK, Russell CD, Grauso NL, Brolin RE. Lipoprotein lipase regulation by insulin and glucocorticoid in subcutaneous and omental adipose tissues of obese women and men. J Clin Invest. 1993;92(5):2191-2198. doi:10.1172/JCI116821.

35. Koliwad SK, Kuo T, Shipp LE, et al. Angiopoietin-like 4 (ANGPTL4, fasting-induced adipose factor) is a direct glucocorticoid receptor target and participates in glucocorticoid-regulated triglyceride metabolism. J Biol Chem. 2009;284(38):2559325601. doi:10.1074/jbc.M109.025452.

36. Wang B, Wood IS, Trayhurn P. Dysregulation of the expression and secretion of inflammation-related adipokines by hypoxia in human adipocytes. Pflugers Arch. 2007;455(3):479-492. doi:10.1007/s00424-007-0301-8.

37. González-Muniesa P, de Oliveira C, Pérez de Heredia F, Thompson MP, Trayhurn P. Fatty acids and hypoxia stimulate the expression and secretion of the adipokine ANGPTL4 (angiopoietin-like protein 4/ fasting-induced adipose factor) by human adipocytes. J Nutrigenet Nutrigenomics. 2011;4(3):146-153. doi:10.1159/000327774.

38. Lu B, Moser A, Shigenaga JK, Grunfeld C, Feingold KR. The acute phase response stimulates the expression of angiopoietin like protein 4. Biochem Biophys Res Commun. 2010;391(4):1737-1741. doi:10.1016/j.bbrc.2009.12.145.

39. Tjeerdema N, Georgiadi A, Jonker JT, et al. Inflammation increases plasma angiopoietin-like protein 4 in patients with the metabolic syndrome and type 2 diabetes. BMJ open diabetes Res care. 2014;2(1):e000034. doi:10.1136/bmjdrc-2014000034.

40. Quagliarini F, Wang Y, Kozlitina J, et al. Atypical angiopoietin-like protein that regulates ANGPTL3. Proc Natl Acad Sci U S A. 2012;109(48):19751-19756. doi:10.1073/pnas.1217552109.

41. Yoshida K, Shimizugawa T, Ono M, Furukawa H. Angiopoietin-like protein 4 is a potent hyperlipidemia-inducing factor in mice and inhibitor of lipoprotein lipase. $\mathrm{J}$ Lipid Res. 2002;43(11):1770-1772. doi:10.1194/jlr.C200010-JLR200.

42. Shimizugawa T, Ono M, Shimamura M, et al. ANGPTL3 decreases very low density lipoprotein triglyceride clearance by inhibition of lipoprotein lipase. J Biol Chem. 2002;277(37):33742-33748. doi:10.1074/jbc.M203215200.

43. Wang Y, Quagliarini F, Gusarova V, et al. Mice lacking ANGPTL8 (Betatrophin) manifest disrupted triglyceride metabolism without impaired glucose homeostasis. Proc Natl Acad Sci U S A. 2013;110:16109-16114. doi:10.1073/pnas.1315292110.

44. Fu Z, Yao F, Abou-Samra AB, Zhang R. Lipasin, thermoregulated in brown fat, is a novel but atypical member of the angiopoietin-like protein family. Biochem Biophys Res Commun. 2013;430(3):1126-1131. doi:10.1016/j.bbrc.2012.12.025.

45. van Raalte DH, Brands M, Serlie MJ, et al. Angiopoietin-like protein 4 is differentially regulated by glucocorticoids and insulin in vitro and in vivo in healthy humans. Exp Clin Endocrinol Diabetes. 2012;120(10):598-603. doi:10.1055/s-0032-1321864. 
46. Kaplan R, Zhang T, Hernandez M, et al. Regulation of the angiopoietin-like protein 3 gene by LXR. J Lipid Res. 2003;44(1):136-143. http://www.ncbi.nlm.nih.gov/pubmed/12518032. Accessed November 25, 2013.

47. Lee J, Hong S-W, Park SE, et al. AMP-activated protein kinase suppresses the expression of LXR/SREBP-1 signaling-induced ANGPTL8 in HepG2 cells. Mol Cell Endocrinol. 2015;414:148-155. doi:10.1016/j.mce.2015.07.031.

48. Nidhina Haridas PA, Soronen J, Sädevirta S, et al. Regulation of Angiopoietin-Like Proteins (ANGPTLs) 3 and 8 by Insulin. $J$ Clin Endocrinol Metab. 2015;100(10):E1299-307. doi:10.1210/jc.2015-1254. 
Table 1 : Patient Characteristics. Data are presented as mean $\pm \mathrm{SD}$, except ${ }^{1}$ which is presented as range. Fasting glucose was determined by the Laboratory of Clinical Chemistry and Haematology, Rijnstate Hospital, Arnhem, The Netherlands. For plasma triglyceride and free fatty acids: $n=59$.

\section{Patient characteristics $(n=76)$}

\section{Gender, $n$ males (\%)}

$35(46 \%)$

For females: menstrual state

Premenopausal, $n$ females (\% of total females)

$15(37 \%)$

Postmenopausal, $n$ females ( $\%$ of total females)

$12(29 \%)$

Unknown, $n$ females (\% of total females)

$14(34 \%)$

Diagnosed with type 2 diabetes, $n$ cases (\%)

$30(39 \%)$

Medication:

Insulin injection, $n$ patients ( $\%$ of total diabetics)

$1(3 \%)$

Metformin, $n$ patients ( $\%$ of total diabetics)

$16(53 \%)$

Glimepiride (sulfonylurea), $n$ patients (\% of total diabetics)

$4(13 \%)$

Unknown, $n$ patients ( $\%$ of total diabetics)

$2(7 \%)$

Combination therapy

Metformin + Glimepiride (sulfonylurea), $n$ patients ( $\%$ of total diabetics)

$3(10 \%)$

Metformin + Gliclazide (sulfonylurea), $n$ patients ( $\%$ of total diabetics)

$4(13 \%)$

\begin{tabular}{|c|c|}
\hline Age at surgery, range in years ${ }^{1}$ & $21-69$ \\
\hline Weight, kg & $129.0 \pm 18.9$ \\
\hline BMI, $\mathrm{kg} / \mathrm{m}^{2}$ & $42.7 \pm 5.3$ \\
\hline Waist circumference, $\mathbf{c m}$ & $133.6 \pm 15.4$ \\
\hline Fasting plasma glucose, $\mathrm{mmol} / \mathrm{L}$ & $7.8 \pm 2.8$ \\
\hline Fasting plasma triglycerides, $\mathrm{mmol} / \mathrm{L}$ & $2.4 \pm 2.1$ \\
\hline Fasting plasma free fatty acids, $\mathrm{mmol} / \mathrm{L}$ & $0.90 \pm 0.34$ \\
\hline
\end{tabular}




\section{Figure Legends}

Figure 1. mRNA levels of ANGPTL3, ANGPTL4 and ANGPTL8 are not correlated in human liver.

(A) Correlation between liver PPARA mRNA levels and liver ANGPTL4 mRNA levels in liver material obtained from patients undergoing bariatric surgery $(\mathrm{n}=59)$. (B) Correlation between liver ANGPTL4 mRNA levels and liver ANGPTL3 and ANGPTL8 mRNA levels in liver material obtained from patients undergoing bariatric surgery $(\mathrm{n}=59)$. (C) Correlation heat map of ANGPTL4, ANGPTL3, ANGPTL8, and PPARA mRNA levels, plasma ANGPTL4 concentrations, fasting plasma glucose levels, plasma levels of non-esterified fatty acids (NEFA) and plasma triglyceride (TG) concentration $(n=59)$. Significant correlations are indicated with a black box. Correlations were analyzed by Spearman's R, p-values of $<0.05$ were considered statistically significant.

Figure 2. Expression levels of ANGPTL4 are highest in the subcutaneous adipose tissue. (A) ANGPTL4 mRNA levels in residual material of the omental, subcutaneous and mesenteric adipose tissue depots obtained from patients undergoing bariatric surgery $(n=75)$. (B) $L P L$ mRNA levels in residual material of the omental, subcutaneous and mesenteric adipose tissue depots obtained from patients undergoing bariatric surgery $(n=75)$. (C) Correlation between ANGPTL4 mRNA levels in the subcutaneous adipose tissue (AT) and ANGPTL4 mRNA levels in the omental and mesenteric adipose tissue depots $(\mathrm{n}=75)$. A.U. signifies Arbitrary Units (D) Correlation between $L P L$ mRNA levels in the subcutaneous adipose tissue (AT) and $L P L$ mRNA levels in the omental and mesenteric adipose tissue depots $(\mathrm{n}=75)$. A.U. signifies Arbitrary Units. Differences in ANGPTL4 and LPL mRNA levels between different adipose tissue depots were analyzed by a Kruskal-Wallis ANOVA followed by a Dunn's test for multiple comparisons. ** $\mathrm{p}<0.01$, *** $\mathrm{p}<0.001$, **** $\mathrm{p}<0.0001$. Correlations between ANGPTL4 and LPL mRNA levels between different adipose tissue depots were analyzed by Spearman's R, p-values of $<0.05$ were considered statistically significant.

Figure 3. Expression levels of ANGPTL4 and LPL are positively correlated in the omental and subcutaneous adipose tissue.

(A) Correlation between ANGPTL4 mRNA levels and LPL mRNA levels in the omental adipose tissue (AT) depots of patients undergoing bariatric surgery $(\mathrm{n}=75)$. (B) Correlation between ANGPTL4 mRNA levels and LPL mRNA levels in the subcutaneous adipose tissue (AT) depots of patients undergoing bariatric surgery $(\mathrm{n}=76)$. (C) Correlation between ANGPTL4 mRNA levels and LPL mRNA levels in the mesenteric adipose tissue depots of patients undergoing bariatric surgery (n=76). (D) Correlation heat map of ANGPTL4, LPL and $P P A R G$ mRNA levels in the omental, subcutaneous and mesenteric adipose tissue depots, plasma ANGPTL4 concentrations, fasting plasma glucose levels, plasma levels of nonesterified fatty acids (NEFA) and plasma triglyceride (TG) levels ( $\mathrm{n}=76$ for gene expression correlations in the omental adipose tissue, $\mathrm{n}=75$ for gene expression analyses in the subcutaneous and mesenteric adipose tissue depots, and $n=59$ for correlations with plasma parameters). Significant correlations are indicated with a black box. Correlations were analyzed by Spearman's R, p-values of $<0.05$ were considered statistically significant. 
Figure 4. Protein levels of LPL and ANGPTL4 are negatively correlated in subcutaneous adipose tissue.

(A) Representative Western blots for LPL and ANGPTL4 in the subcutaneous adipose tissue depot of nine patients undergoing bariatric surgery (see Supplemental Figure 4 for the Western blots of all patients). (B) Correlation of protein levels of LPL and ANGPTL4 in the subcutaneous adipose tissue of patients undergoing bariatric surgery, as determined by the quantification of Western blots $(n=54)$. Correlation was analyzed by Spearman's $\mathrm{R}, \mathrm{p}$-values of $<0.05$ were considered statistically significant.

Figure 5. ANGPTL4 mRNA levels are higher in the fasted than the post-prandial state in subcutaneous adipose tissue.

mRNA expression levels of ANGPTL4, ANGPTL8 and LPL in subcutaneous adipose tissue of 72 subjects for which baseline fat biopsies were available. Biopsies were taken after an overnight fast, and 4 hours after consumption of a mixed meal consisting of $76.3 \mathrm{~g}$ carbohydrates, $17.6 \mathrm{~g}$ protein, and $60.0 \mathrm{~g}$ fat (post-prandial). Expression levels were determined using Affymetrix microarray analysis. A single line represents one subject. ANGPTL4 mRNA levels were $38 \%$ higher in the fasted state compared to the post-prandial state (Paired Student's t-test, $\mathrm{P}<1 * 10^{-15}$ ). ANGPTL 8 mRNA levels were $64 \%$ lower in the fasted state compared to the post-prandial state (Paired Student's t-test, $\mathrm{P}<1 * 10^{-22}$ ). $L P L$ mRNA levels were not significantly different between the fasted and post-prandial state. 
Figure 1
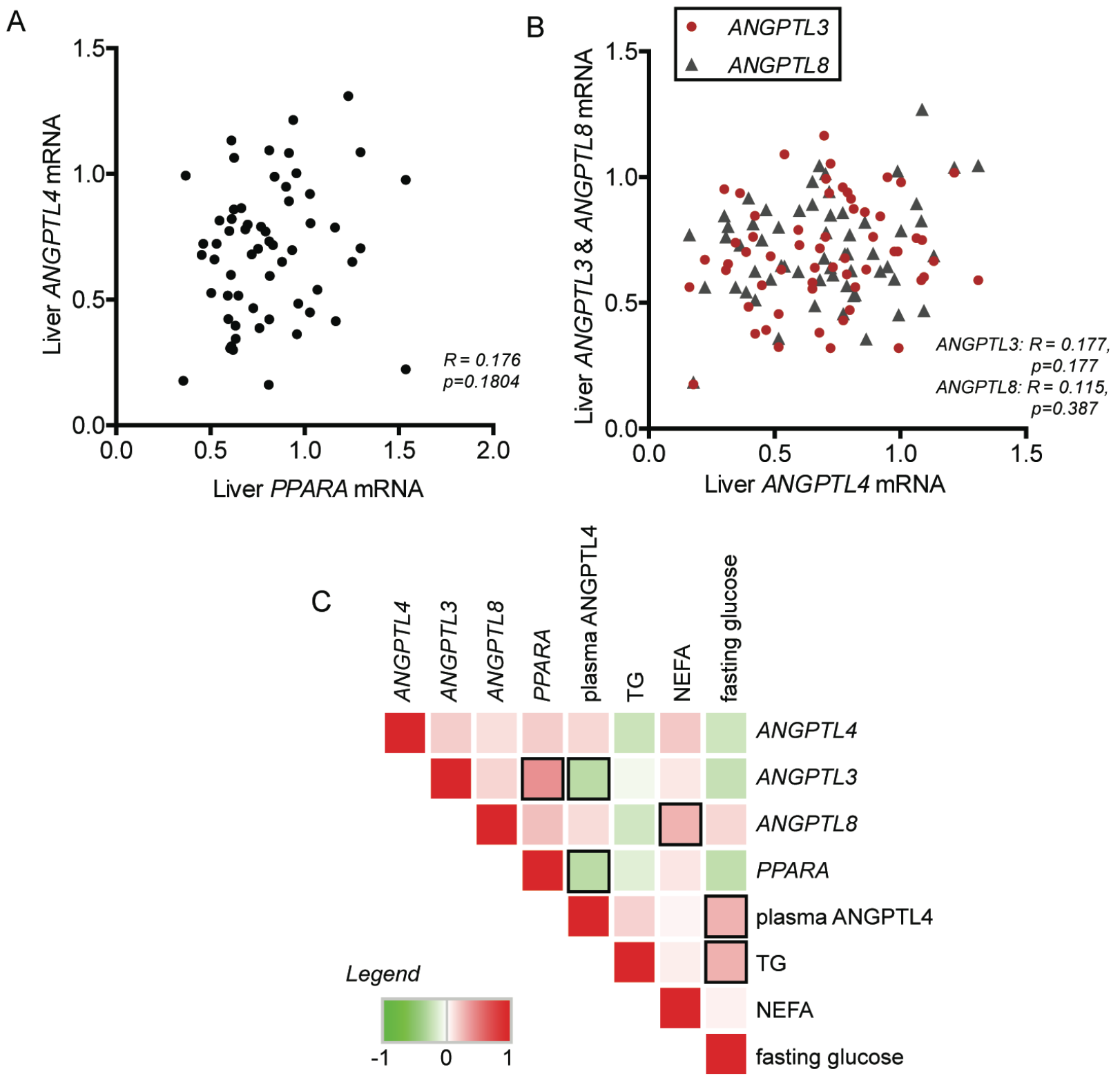
Figure 2

A

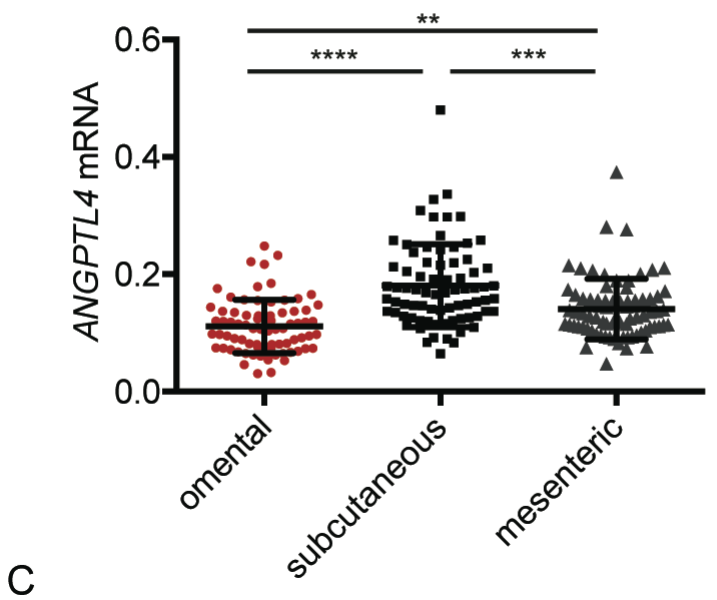

C

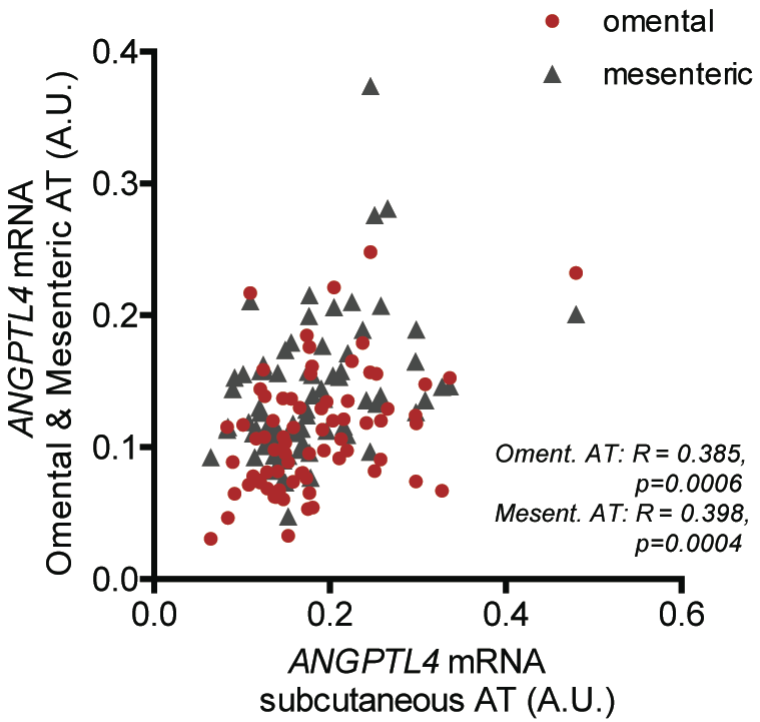

B

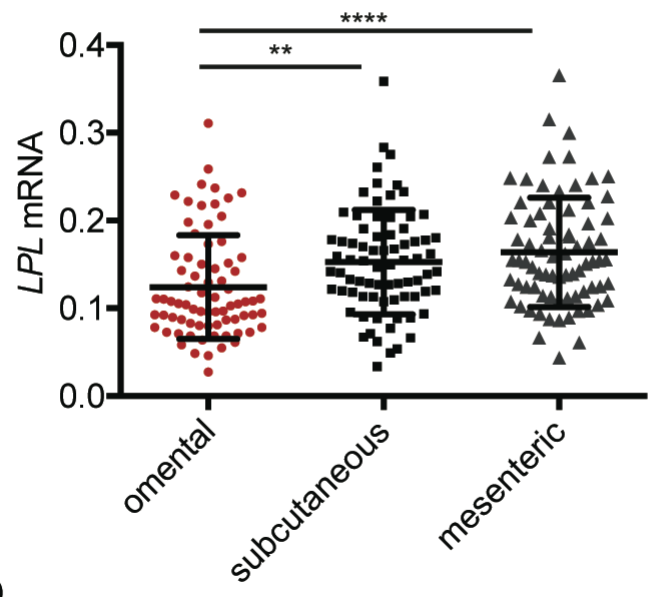

D

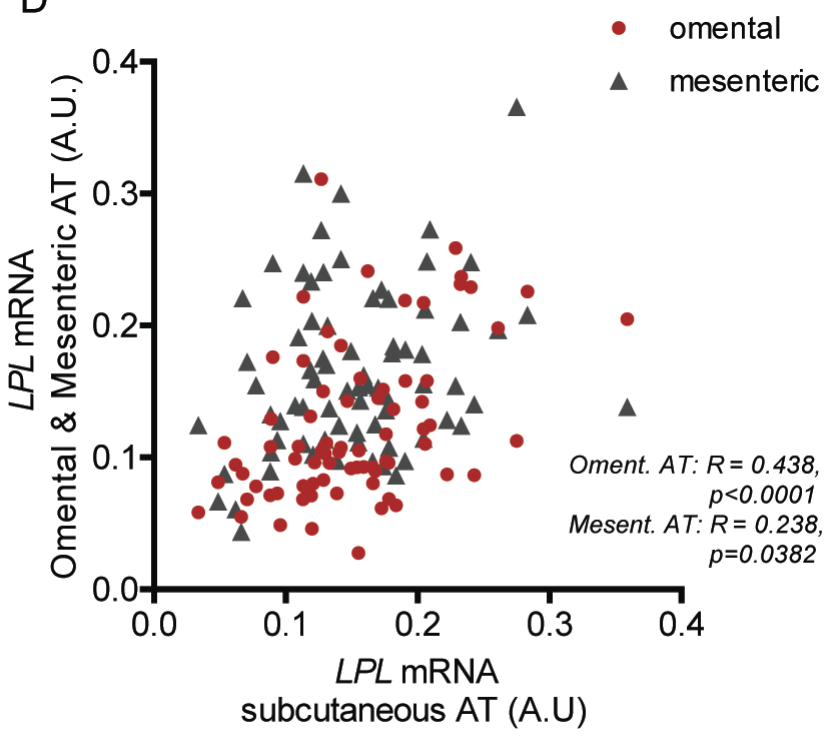


Figure 3
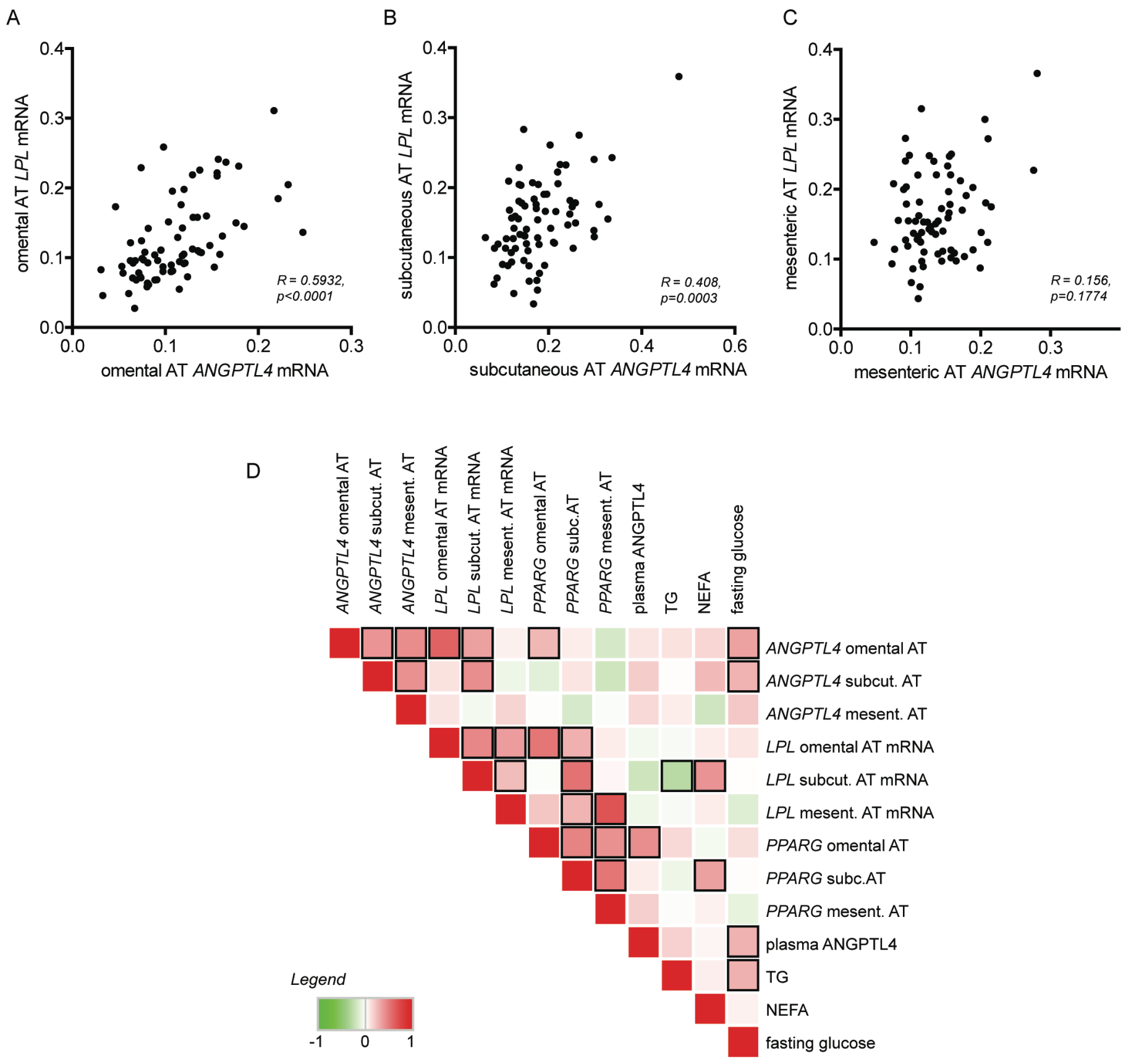
Figure 4

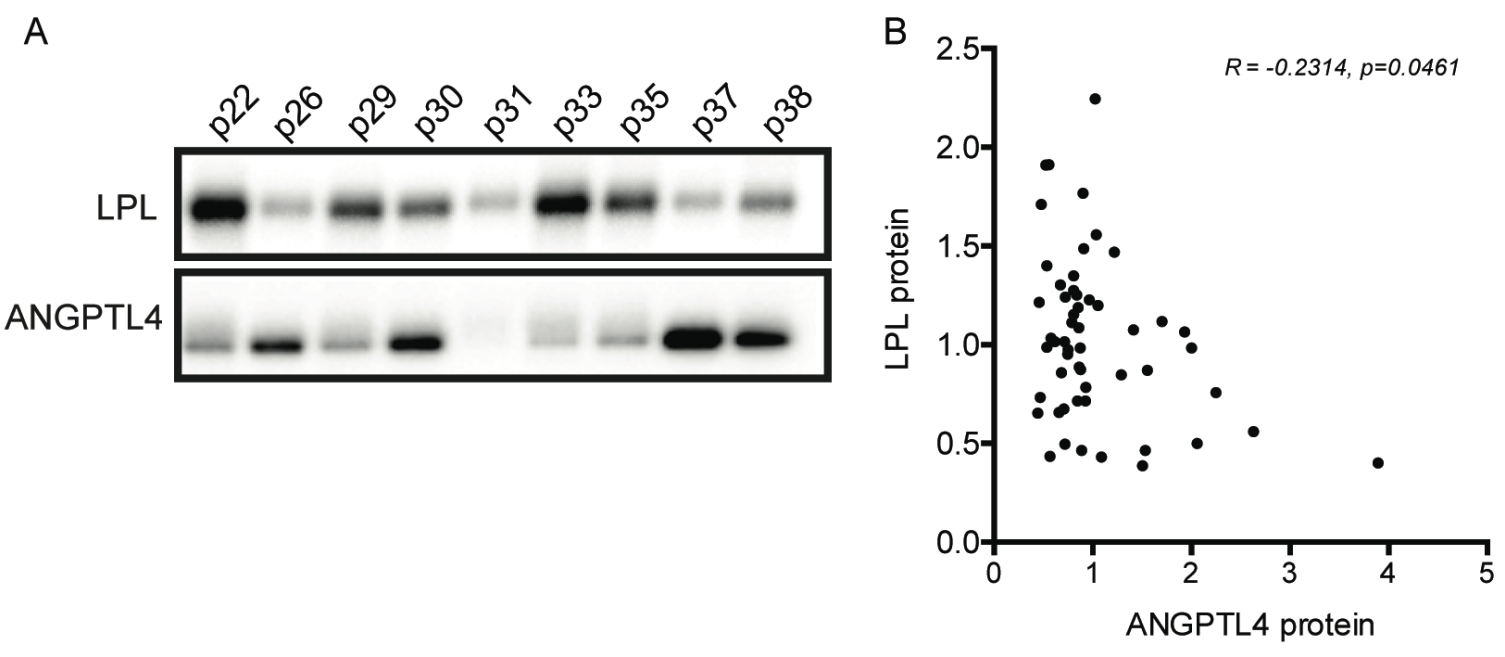


Figure 5
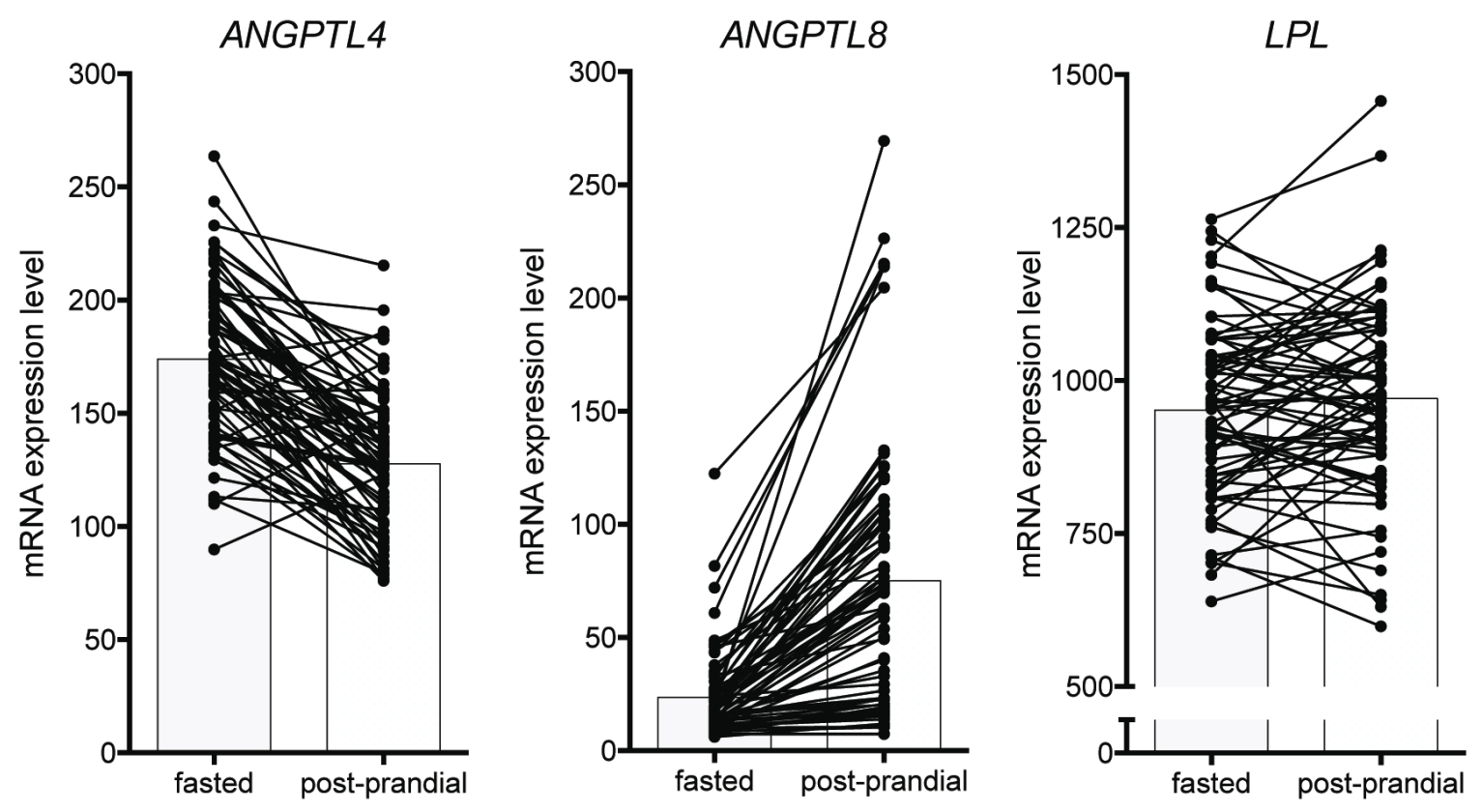